\title{
DEVELOPMENT OF AUGMENTED REALITY PIPELINE VISUALISER (ARPV) APPLICATION FOR VISUALISING UNDERGROUND WATER PIPELINE
}

\author{
Li Meng Cheng ${ }^{1}$, Shahabuddin Amerudin ${ }^{2,}$, Zainab Mohamed Yusof ${ }^{3}$ \\ ${ }^{1,2}$ Faculty of Built Environment and Surveying, Universiti Teknologi Malaysia, Johor Bahru, Malaysia - (mcli2@live.utm.my, \\ shahabuddin@utm.my) \\ ${ }^{3}$ School of Civil Engineering, Universiti Teknologi Malaysia, Johor Bahru, Malaysia - (zainabyusof@utm.my)
}

KEY WORDS: Android, Unity3D, Mapbox, Underground Utility Locating, ARPV

\begin{abstract}
:
Underground utilities locating is an important process to identify buried utility facilities for inspection and maintenance purposes. The process involves the use of underground utility maps and detecting devices to identify utilities as well as paints to mark the positions. However, this practice requires sophisticated devices, and skilful and experienced workers. The whole process takes time and is costly to operate. To solve these difficulties, an easy to use Augmented Reality (AR) mobile application, namely AR Pipeline Visualiser (ARPV) was developed. ARPV has feature for identifying pipelines which allows their attributes to be displayed. Besides, it also comes with colour classification buttons based on the pipelines' attribute values which help users to discriminate pipelines better. ARPV uses spatial data in GeoJSON format which is hosted on Mapbox's online database. ARPV is developed using Unity3D engine and Mapbox SDK for Unity. ARPV utilizes smartphone's sensors to determine the location and orientation of the device, then, render the corresponding pipelines graphics on top of the reality scene through camera and screen. A qualitative testing with an engineer from Pejabat Harta Bina, Universiti Teknologi Malaysia shows that the application has an advantage in providing better visualisation and understanding of water pipeline than conventional method.
\end{abstract}

\section{INTRODUCTION}

Hidden and away from the public, underground utility mains served to supply necessities such as water, electricity and natural gas to the public. These infrastructures run underground to keep the city aesthetic and safe, as well as to protect the infrastructure itself (McCarthy, 2011). Despite being buried, the infrastructures are still prone to damage caused by various factors such as pipe degradation, natural disaster and accidental damage during excavation (McCarthy, 2011). Installing and maintaining them underground are complicated as they are buried under the earth. Utility locating serves to locate and mark them. Tools such as utility maps and utility detector are used in the locating process. (Health and Safety Executive, 2014; Pestov, 2018). Utility locating helps to locate the position of underground utility and avoid damaging them during excavation while keeping a safe working environment for the workers at the same time (Health and Safety Executive, 2014).

As a matter of fact, the major cause of telecommunication disruption in Malaysia is due to accidental damage of underground cables (The Sun Daily, 2017). For example, in 2018, multiple fiber cable belonging to Telekom Malaysia Berhad had been damaged accidentally due to excavation in Sentul and Sabak Bernam West, Kuala Lumpur. The incident has disrupted the Internet services and restoration took more than 24 hours (The Star, 2018). Hence, it is vital to make sure that excavation can be carried out at low risk by performing utility locating. This can help to prevent unnecessary losses. Conventional approach of locating underground utility involves the use of utility maps together with various detecting equipment (Health and Safety Executive, 2014; Pestov, 2018). The operator then marks the location on the ground upon detecting underground asset. The markings serve as the guidance for the excavator during excavation (Behzadan and Kamat, 2009).

The quality of the locate work, however, may deteriorate due to less experienced or careless operator (Behzadan and Kamat, 2009; Pestov, 2018). Besides, other factors such as density of utility, ground condition and depth of utility may also contribute to the inaccuracy of locate work (Behzadan and Kamat, 2009; Jaw and Hashim, 2014). If taking into time measurements, the overall process from understanding map to marking the location of assets takes up time depending on the job size before an excavation can start (Behzadan and Kamat, 2009). In the campus of Universiti Teknologi Malaysia (UTM) in Johor Bahru, Malaysia however, thing is done differently.

Locations of underground water pipeline are identified by senior employees of Pejabat Harta Bina (PHB). PHB is a department responsible in maintaining the assets in campus since 1973 (Office of Asset, 2017). Based on interview with an engineer and representative of $\mathrm{PHB}$, since the construction of new campus in 1985, employees of PHB, have involved closely with the construction and maintenance activities. Hence, these senior employees can still identify the position of underground pipeline inside the campus without any tools. As the years go by, these employees will be retiring soon and at the time, new employees may not be able to identify the location of underground pipelines. Therefore, measure needs to be taken before the time comes.

To overcome the shortcoming of conventional approach for locating utilities, researchers make use of satellite positioning together with Augmented Reality (AR) technology and various

\footnotetext{
* Corresponding author
} 
sensors on a mobile device to locate as well as visualize utilities in real world scenes (Li et al., 2018). The technology of current mobile device makes AR even more practical to be used in this purpose. With its light weight and small size, it contains all necessary components to operate AR ( $\mathrm{Li}$ et al., 2018). AR is a technology that is capable of overlaying information such as pictures, video and context on top of the real-world scene captured via camera and streamed through a display (Hosch, 2018).

Through sensors and computing, a device's real time orientation and location can be determined. This information is then used to position generated graphics in their corresponding real-world position (Li et al., 2018). The technology can provide faster, accurate and vivid localization and representation of underground pipelines (Schall et al., 2010). A study conducted by Meemim Inc. (2018) on their own AR solution found that their product can helps to improve the process of a locating job compared with conventional method. Time spent for each locating job is reduced by up to $50 \%$. In average, a locator can save up to 12-20 hours per month. Moreover, other benefits claimed by Meemim Inc. (2018) include improved safety, accuracy and ease of locating job. Besides, the solution also enhances the understanding of locators' environment and increase their situational awareness (Pestov, 2018; Rees, 2018).

Another AR solution developed by Schall et al. (2010) found that AR solution provides a much practical yet handy solution for underground utilities inspection. He agrees that both time and money can be saved by using AR solution for locating underground utilities. The solution namely Vidente works by fetching GIS data from a server and render it on a device which creates an illusion that the rendered graphics is overlay on top of the real world. He also found that the ability to render mains in different color coding is also highly desired by the industry. Similar technique is utilized by $\mathrm{Li}$ et al. (2018) in developing AR solution for visualizing underground pipeline.

Li et al. (2018) stored their spatial data in a server then transmit it to the device via request. The data received will then be converted into coordinate of Unity3D and overlain on the scene. However, Differential Global Positioning System (DGPS) technique is implemented by $\mathrm{Li}$ et al. (2018) to improve the accuracy of positioning. On top of that, scene recognition technique is also implemented to overcome positional inaccuracy.

This project is aim to developed an AR solution specifically for the use of PHB, UTM. It serves to visualize and locate underground water pipelines in the campus of UTM. Instead of developing from scratch, this project utilized and modified the prefabrication provided in Mapbox SDK for Unity. The paper is organized in the following order: first, introduction in Section 1 , the concept, system architecture design and development of the solution in Section 2. The results and discussions are presented in Section 3 and conclusion of the project with some future work remarks in Section 4.

\section{METHODOLOGY}

\subsection{User Requirement Analysis}

Initial phase of the approach involves defining the problem statement, identifying project aim and objectives, scheduling project activities, literature review as well as to perform User Requirement Analysis (URA). Meetings with users were done to understand their problems and desires for the solution. Possible limitations of the project were also identified, discussed and negotiated in the process. Functional and nonfunctional requirements of the application were then defined, described and agreed by both parties. The agreed requirements are as shown in Table 1 and Table 2.

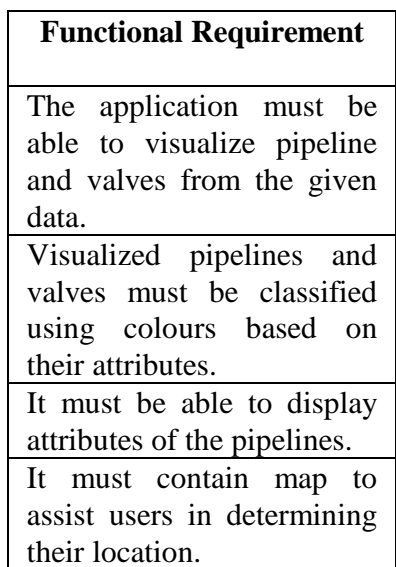

Table 1. Functional requirement of the application

\begin{tabular}{|l|}
\hline \multicolumn{1}{|c|}{$\begin{array}{c}\text { Non-functional } \\
\text { Requirement }\end{array}$} \\
\hline $\begin{array}{l}\text { Performance of the device } \\
\text { shall be able to work at least } \\
30 \text { minutes continuously to } \\
\text { meet the demand of the work } \\
\text { nature. }\end{array}$ \\
\hline $\begin{array}{l}\text { Reaction time of the } \\
\text { application during } \\
\text { initializing shall fall within } \\
20 \text { seconds. }\end{array}$ \\
\hline
\end{tabular}

Table 2. Non-functional requirement of the application

\subsection{ARPV System Architecture Design}

As given by Mapbox, ARPV is based on a two-tier system architecture with a client tier and server tier as shown in Figure 1. The client-tier is a smartphone with ARPV application installed. In this tier, the data which in GeoJSON format is requested and retrieved from server tier through Internet connection. The data is then processed and visualized in $A R$ view on the client tier. At the same time, a raster map style is also downloaded for the Map view in ARPV.

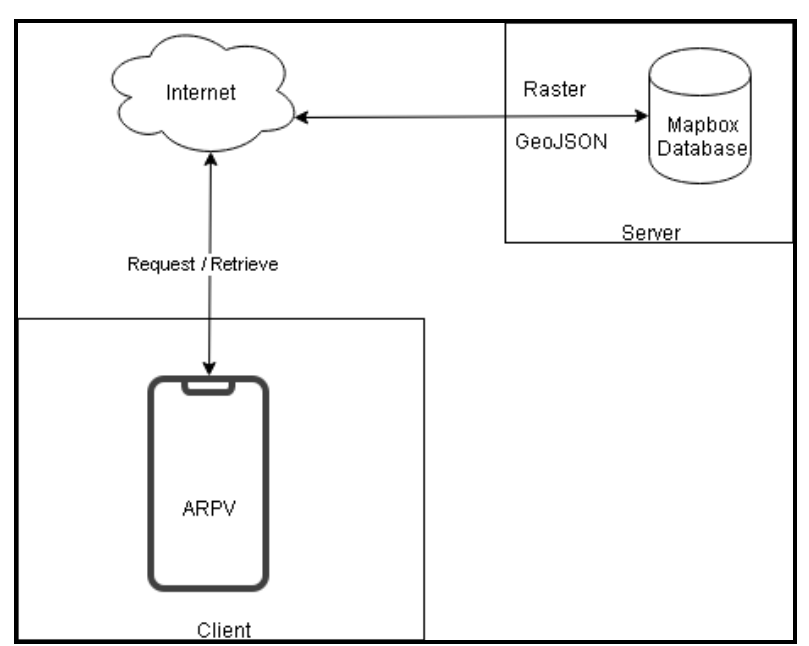

Figure 1. ARPV system architecture design 


\subsection{ARPV Workflow Design}

The workflow of ARPV is arranged as in Figure 2 whereas a simplified workflow of ARPV with UI corresponding to each action is shown in Figure 3.

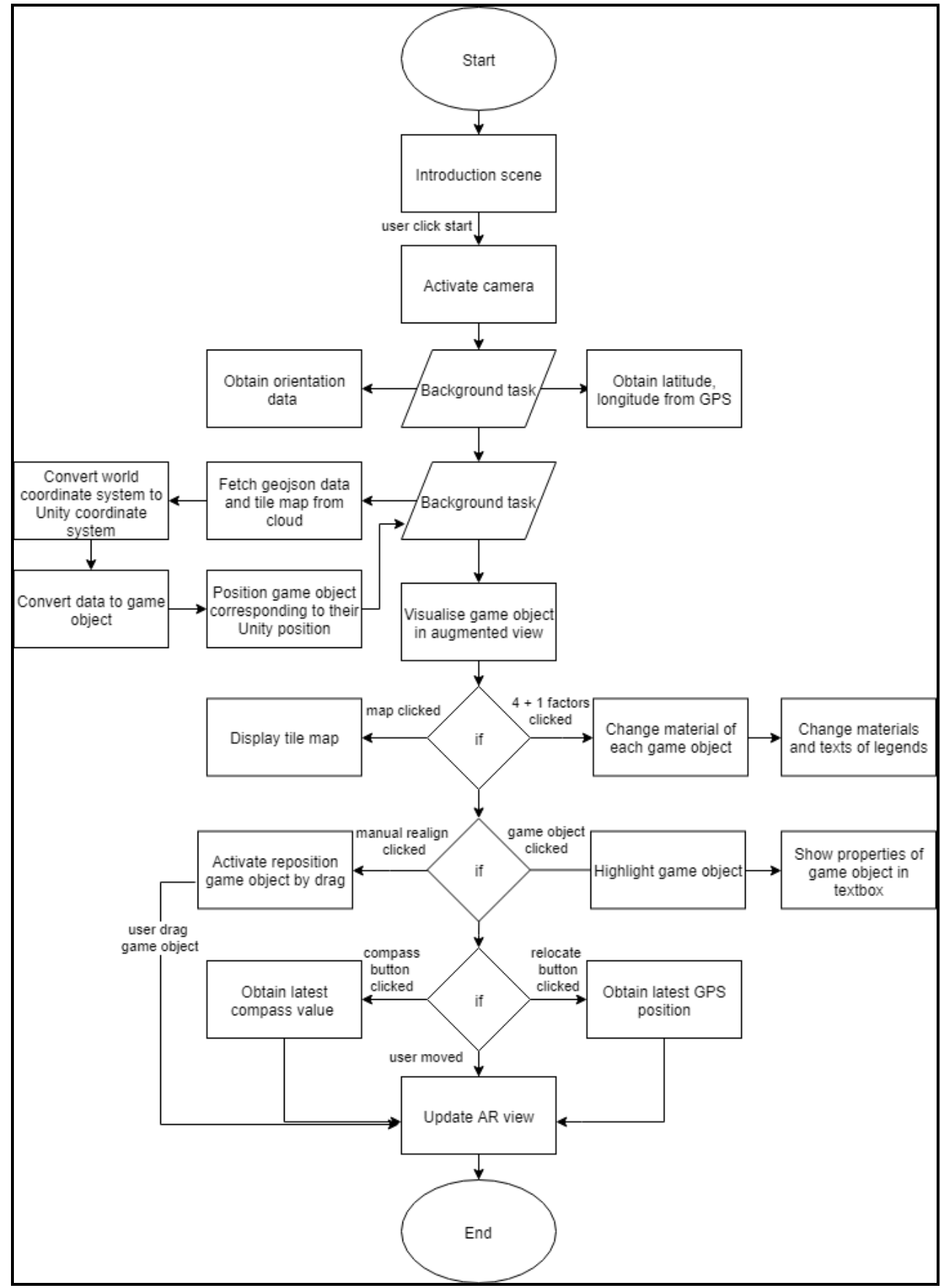

Figure 2. Detailed workflow of ARPV

The AR view of ARPV provides the capability to visualize data in their corresponding real-world position through device's screen. The data, originally in GeoJSON format and WGS 84 coordinate system is fetched from Mapbox's database, parsed and converted into Unity's game object of Unity's coordinates. Each feature is translated into one game object in the process and they are positioned in their respective location in Unity's environment as shown in Figure 4.
Then, through the device's location sensor, the coordinate received is also converted into Unity's coordinate. By using this method, the AR Camera can be aligned to its position in the Unity's environment together with all the pipeline converted game objects. The compass's reading is also obtained during the process to determine the heading of the AR Camera as shown in Figure 5. 
The International Archives of the Photogrammetry, Remote Sensing and Spatial Information Sciences, Volume XLII-4/W16, 2019 6th International Conference on Geomatics and Geospatial Technology (GGT 2019), 1-3 October 2019, Kuala Lumpur, Malaysia

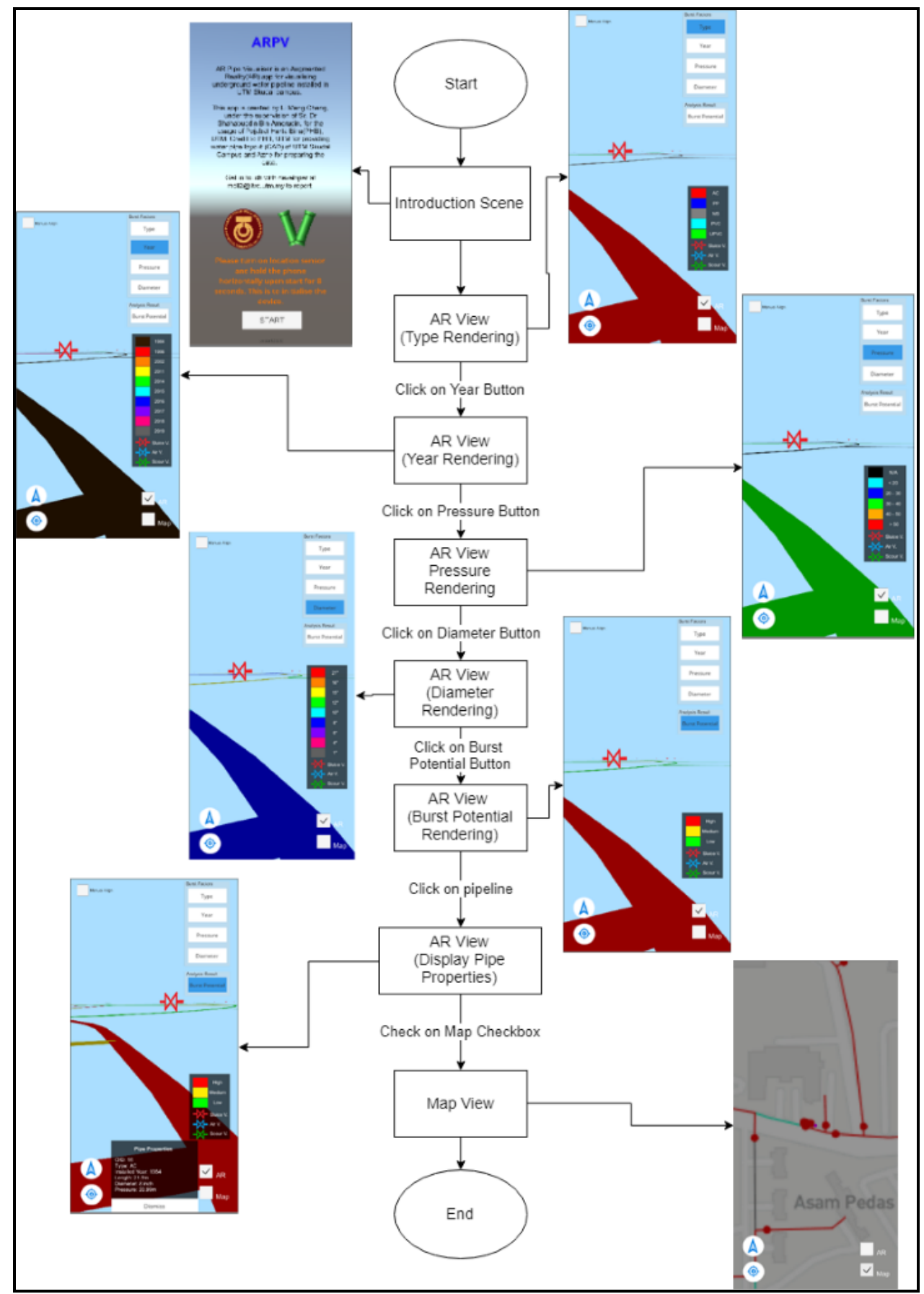

Figure 3. Simplified workflow of ARPV with UI

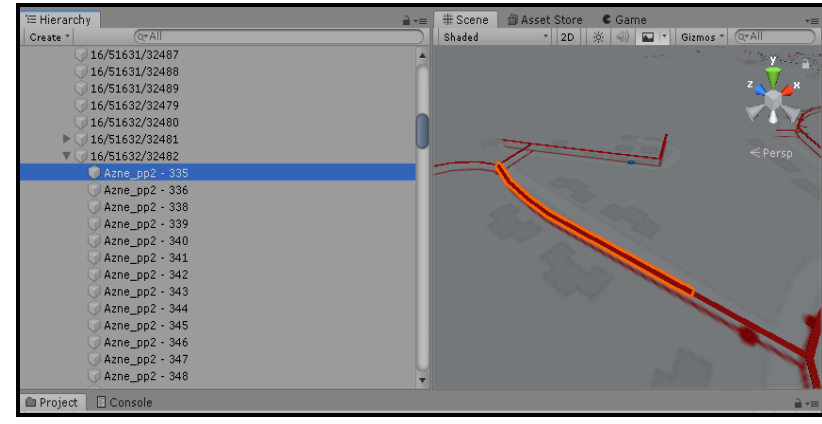

Figure 4. Game objects converted from GeoJSON in their respective location in Unity environment

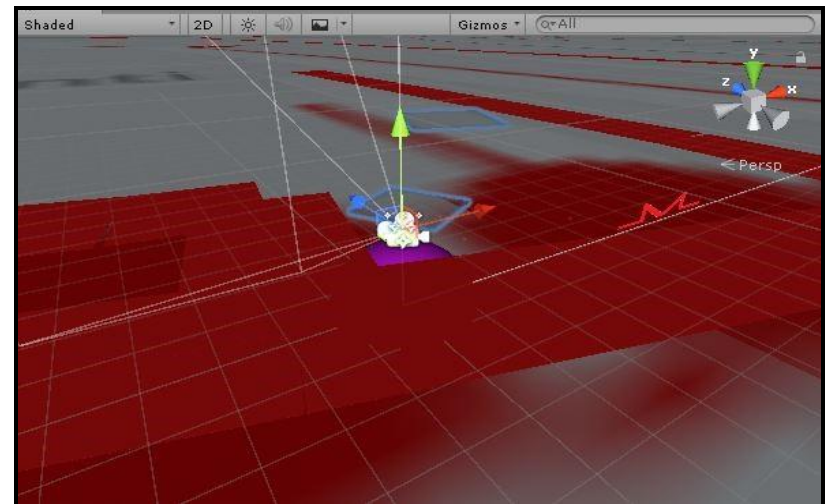

Figure 5. Game objects and AR camera in Unity3D Editor 


\subsection{Logical Data Design}

The spatial extent of the data used in this development covers the whole campus of UTM, Johor. The total length of underground water pipelines is $60.2 \mathrm{~km}$. The logical design of data as shown in Table 3 consists of water pipeline (line) and valves (point) in WGS84 coordinate system; shapefile format.

\begin{tabular}{|c|c|c|}
\hline Feature & Name & Attributes \\
\hline Line & Pipeline & OID (INT) \\
& & Pipe_Type (TXT) \\
& & Year (INT) \\
& & Diameter (INT) \\
& & Pipe_Length (FLOAT) \\
& & Pressure (FLOAT) \\
& & Burst_Potential (INT) \\
\hline Point & Valve & OID (INT) \\
& & Valve_Type(TXT) \\
\hline
\end{tabular}

Table 3. Logical design of data

\subsection{User Interface Design}

The overall design of the UI was modified from Mapbox's prefab and the completed design is as shown in Figure 6 and Figure 7. Unnecessary UI modules were removed from the prefab, and additional modules were added for ARPV's extra features during the design process.

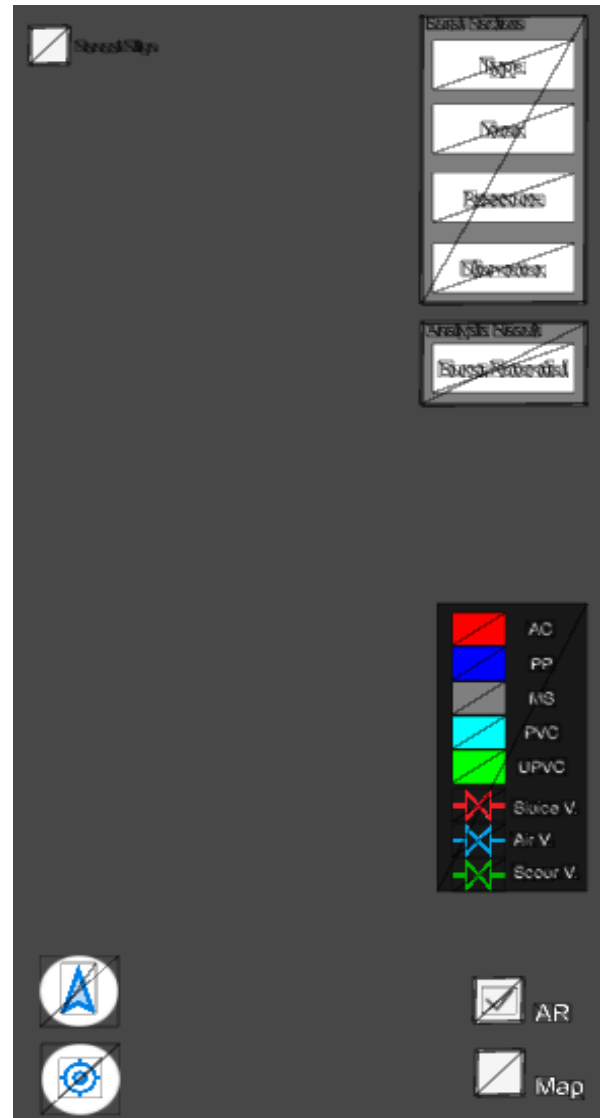

Figures 6. Arrangement UI components of AR view in Unity3D Editor

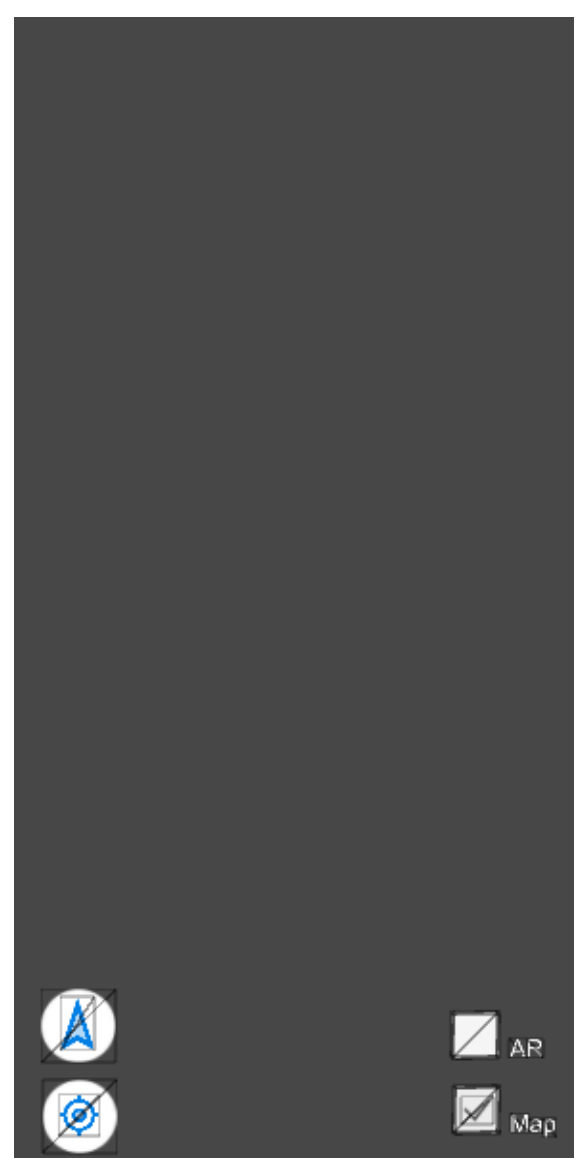

Figures 7. Arrangement UI components of map view in Unity3D Editor

The top left corner of the UI is a checkbox to toggle the manual alignment feature. On the bottom left corner are two buttons for relocate position and re-orientate. Re-orientate button also acts as compass at the same time. Its arrow will rotate when the device rotates. On the top right are five buttons which represents five different colour rendering options. On the bottom right are the checkboxes to toggle AR view or map view. On the bottom centre, a textbox with a dismiss button is designated to display the information of pipelines when triggered.

Above the checkboxes is a canvas showing legends which serve to indicates the meaning of each symbol and colour. In map view, manual alignment checkbox, colour rendering buttons and the canvas of legends are turned off to minimize viewing obstruction. The arrangement and design of each modules has taken into the considerations such as finger-friendly, frequency of usage, minimize the obstruction of view and simplicity (Jessie, 2017).

\subsection{Setting up of Mapbox}

To use Mapbox SDK for Unity, Mapbox account is created for the purpose of storing data and creating map. Data as mentioned in Section 3.6, which are shapefiles are compressed into zip file. This step includes all the shapefiles' extension files. Then, the zipped files are uploaded to Mapbox Studio as tile sets which are in GeoJSON format. 


\subsection{Setting Up of Unity3D}

Development of application uses Unity3D game engine and Mapbox SDK for Unity. Unity3D as shown in Figure 7, is a game engine which is commonly used for developing games and animations (Unity Technologies, 2019). However, with ARCore, Unity3D can be used to create AR apps for Android smartphones. While ARCore is a platform installed on an Android smartphone which allow the device to sense and understand its environment using algorithms and sensors (Google, 2019). With Mapbox SDK for Unity, it allows easy integration of spatial data in Unity3D (Mapbox, 2019). It also provides prefabrications which allow developer to start off without having to develop from scratch. Scripting is performed in Microsoft Visual Studio using C\# programming language.

In Unity3D, the MapID of each tilesets from Mapbox Studio is pasted into a designated field in Map Layers of Unity3D as shown in Figure 7.

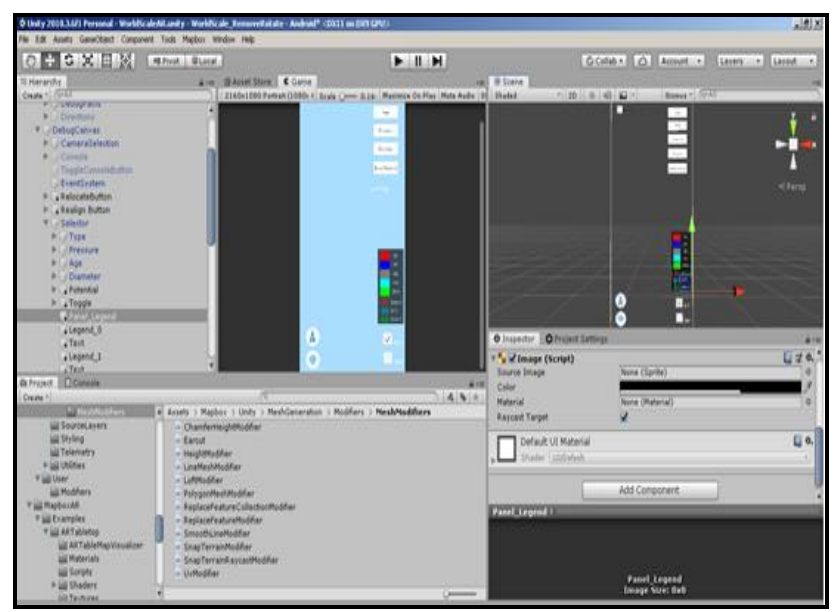

Figure 7. Unity3D integrated with Mapbox SDK for Unity

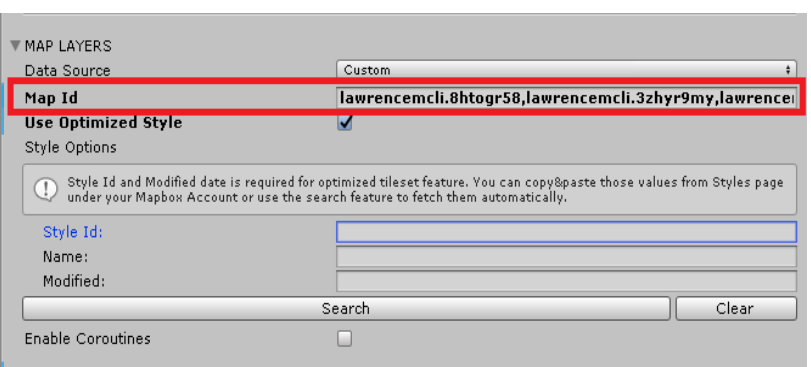

Figure 8. Field for entering MapID of tileset in Unity3D

For map view, a raster map is designed using Mapbox Studio's Styles as shown in Figure 9. Same as tileset, the MapID is also registered in Unity3D. The map will be loaded once the device determines its coordinate. The centre of the map is also set to the coordinate obtained.

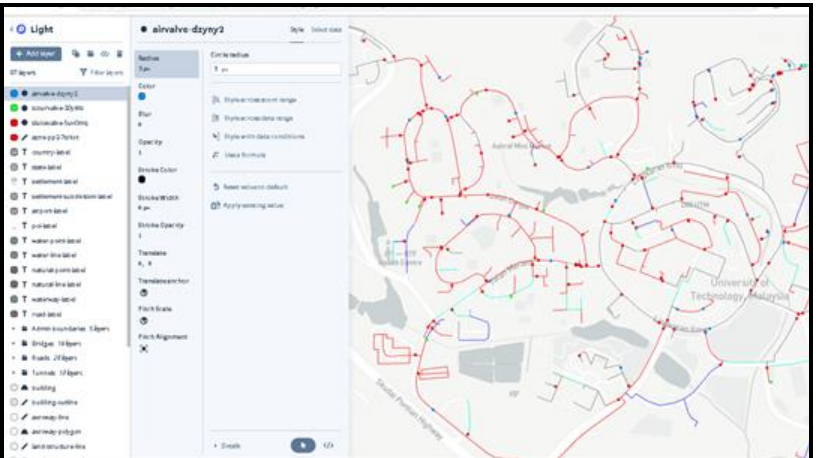

Figure 9. Mapbox's Studio for designing map and manage data

\subsection{Coding}

In Unity3D, each script is attached to a game object which can be triggered. For example, a button to relocate GNSS position is attached with a script which trigger ARPV to relocate its position using the latest captured coordinate value. Using the same concept, each game object of the pipeline is attached with a script, namely "touch.cs" as shown in Figure 10 which trigger them to display their information on a designated textbox upon touch. The information is retrieved from the pre-converted vector data using scripts in Figure 11. Besides, the colour of game objects also can be changed by touching on the designated buttons.

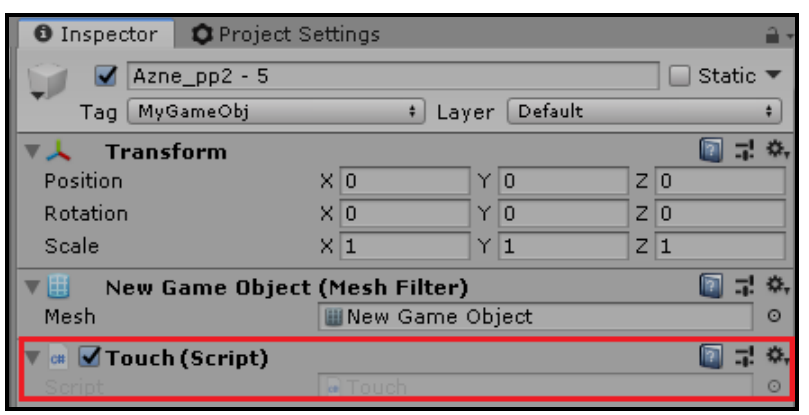

Figure 10. Script attached with one of the game objects which triggering action

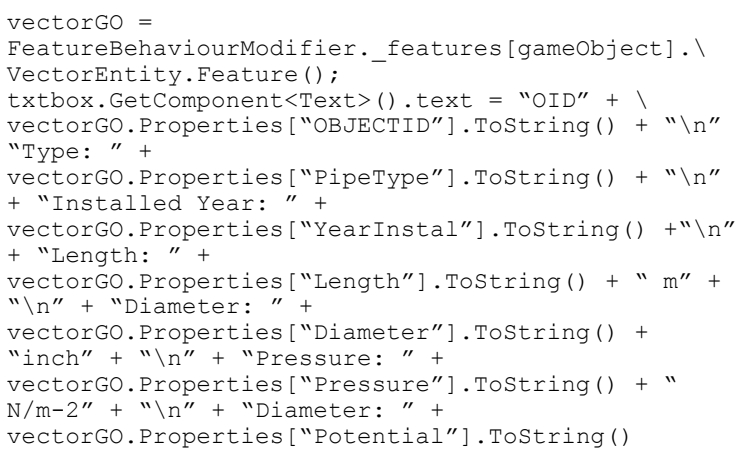

Figure 11. Fragmented script for retrieving attributes of each feature

This functionality could assist users in classifying pipelines visually according to their properties. The fragmented script for this functionality is as shown in Figure 12. Legends are also provided to guide user on the meaning of each colour and symbol. The scripting of legends for type rendering is as shown as Figure 13. Available buttons for colour rendering are 
Type (type of pipes), Year (year of installation), Pressure (water pressure), Diameter (pipeline's diameter), Burst Potential (pipe burst potential).

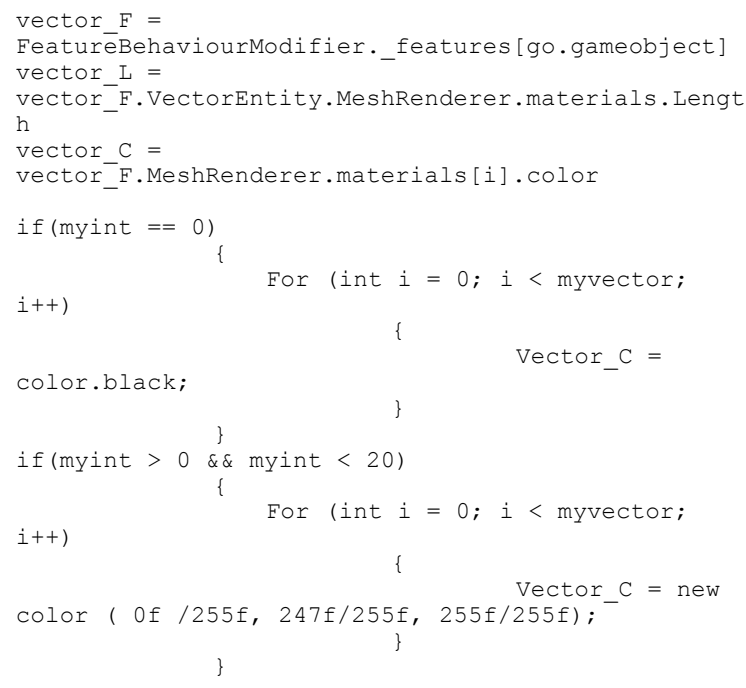

Figure 12. Fragmented script for pipeline colour rendering

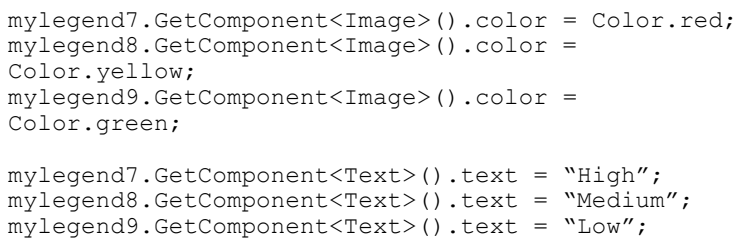

Figure 13. Fragmented script for legends colour rendering and text adding

Colour rendering feature is based on the result obtained from another study which was conducted by Azne Hazira (2019). In the study, potential of pipe burst in UTM using four parameters were determined. These parameters are the type of pipe's material, the year of pipe installation, the pressure reading of pipe and the diameter of pipe. The four parameters were considered as factors in causing pipe burst in UTM. Using these parameters, the value of pipe burst potential for each pipeline were computed. Value "1" indicates low burst potential, "2" indicates medium potential and " 3 " indicates high potential. Therefore, there are five buttons for colour rendering.

\section{EVALUATION AND DISCUSSION}

To evaluate the application, black-box test was performed with an engineer from PHB UTM. The test is of qualitative and is divided into two categories; functional and non-functional tests. Before the test, the engineer was briefed on ARPV application. The test was carried out at a valve within the campus of UTM where pipeline can be seen exposed on the ground surface. Figure 14 to Figure 16 shows the screenshots obtained during the test.

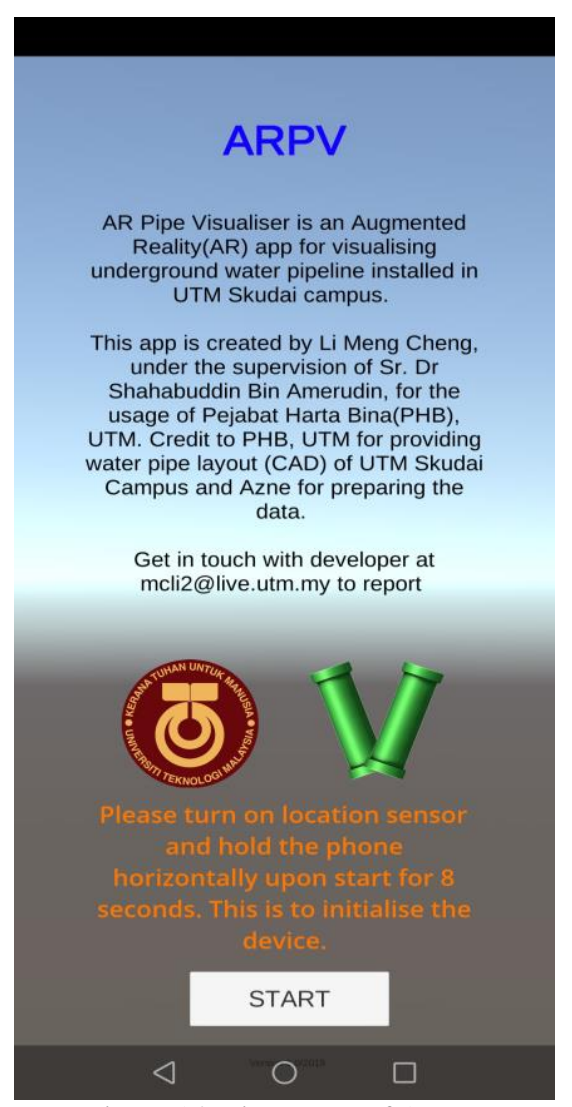

Figure 14. First scene of ARPV

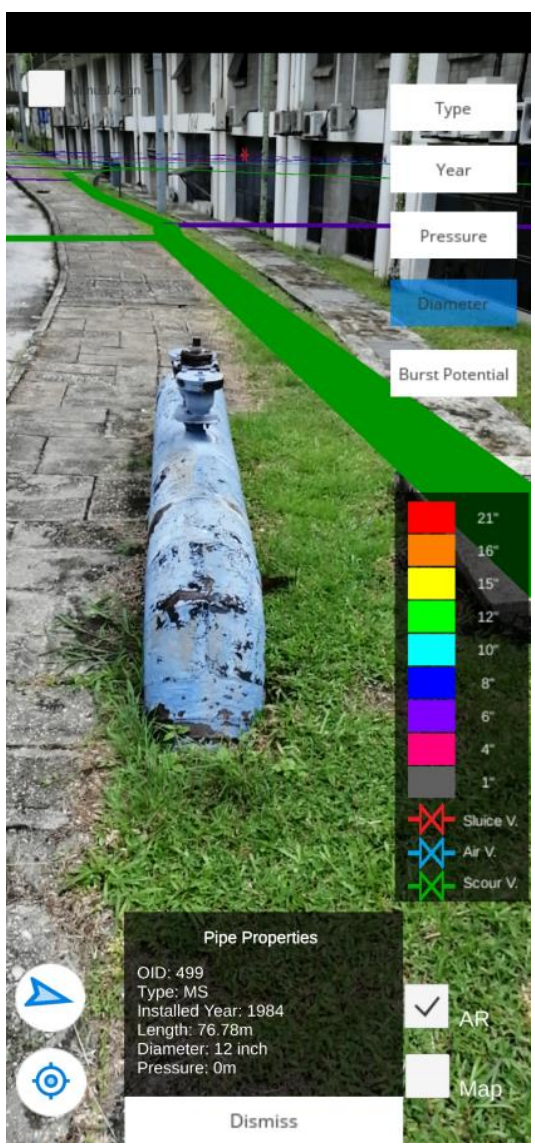

Figure 15. AR view of ARPV 


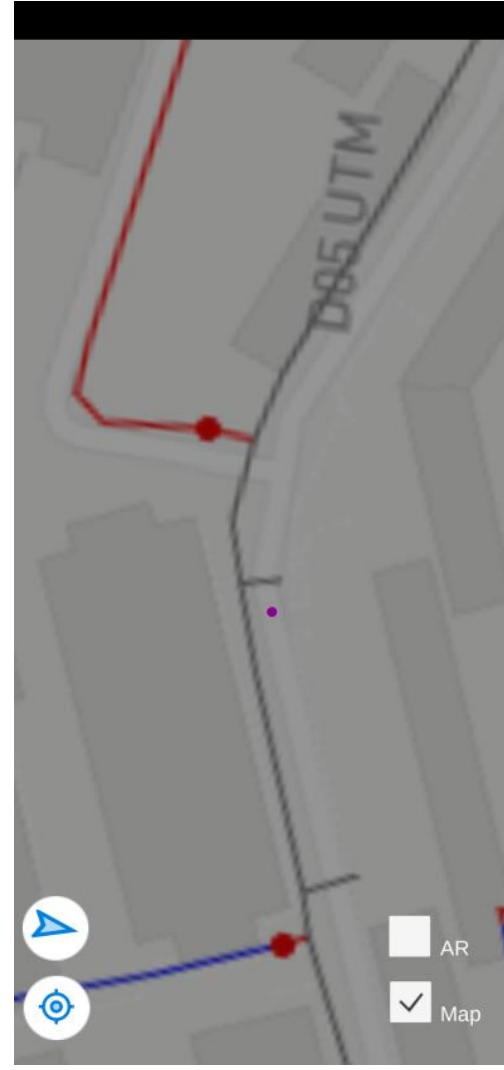

Figure 16. Map view of ARPV

\subsection{Functional Requirement}

The tester has expressed satisfaction in the AR capability to visualize pipeline. AR view can provide the tester with the visualization of pipeline graphics on top of the real-world scene, in their corresponding location. This capability enhanced the tester's surrounding awareness as well as the understanding of pipeline's layout. Moreover, attributes of each pipeline segments can be shown in the designated text box when touched. This capability provides the tester with ability to know the background of each individual pipeline segments. This benefitted the fieldworkers by allowing them to make decision on the spot based on the information provided and shortened the time spent on field. On the other hand, it also can show the current position of the tester on the map which is very beneficial especially for user working at an unfamiliar place.

\subsection{Non-functional Requirement}

For non-functional requirements, the tester was not satisfied with the device used in the test. The device, Honor $8 \mathrm{X}$ experienced freeze during testing and overheat easily after 10 minutes to 15 minutes of usage. Higher end of device with higher processing capability could be used to solve this problem. Alternatively, application logic could be refined to improve the processing efficiency. Conversely, the tester was pleased with the fast visualizing capability of the application which falls within 15 seconds upon starting the application.

Furthermore, the positional accuracy was not good during the test. Typical industrial requirement for positional accuracy requires a maximum of $0.3 \mathrm{~m}$ to be achieved (Behzadan and Kamat, 2009). However, the position of the visualized pipeline was $2 \mathrm{~m}$ away from the actual pipeline. Hence, the accuracy during the test was out of the industrial requirement. The result may be due to multipath signal errors or the actual accuracy of smartphone device which has an average of $4.9 \mathrm{~m}$ in good condition. Reliability of the data visualized is also another factor which may contribute to the inaccurate result.

\section{CONCLUSION}

In this project, the capabilities of AR were successfully leveraged using Mapbox SDK for Unity with the goal to solve the difficulties faced by underground utilities locator as well as PHB, UTM in visualizing underground water pipeline. Due to the limitations of project, there are some existing techniques which could not be implemented in the app such as the scene recognition (Li et. al., 2018) which could greatly improve positional accuracy. Through this project, attributes of each pipelines can be retrieved and utilized to render the color of pipelines. The AR and map view also can assist field workers in understand the layout of underground pipelines while increase their situational awareness at the same time. These advantages can help field workers to obtain the information in short time and make necessary decision on the spot.

For future development, considerations such as improving pipeline graphics, conducting test using various devices, and adding feature for data updating and offline visualization can be taken to enhance the application.

\section{ACKNOWLEDGMENT}

The authors would like to express their appreciation for the financial supports with Vot Number Q.J130000.2527.11H52 from Universiti Teknologi Malaysia and Ministry of Higher Education of Malaysia.

\section{REFERENCES}

Azne Hazira, B. S. (2019). Determination of Potential of Water Pipeline Bursting using Stochastic Approach in GIS. BSc Thesis, Universiti Teknologi Malaysia,

Behzadan, A. H., and Kamat, V. R. (2009)."Interactive augmented reality visualisation for improved damage prevention and maintenance of underground infrastructure," in Construction Research Congress, Seattle, 2009.

Diggelen, F. V., and Enge, P. K. (2015). The World's first GPS MOOC and Worldwide Laboratory using Smartphones.

Google. (2019). ARCore Overview. Retrieved 13/04/2019 from https://developers.google.com/ar/discover/

Health and Safety Executive (2014). Avoiding danger from underground services. Retrieved 01/11/2018 from http://www.hse.gov.uk/pubns/books/hsg47.htm

Jaw, S. W., and Hashim, M. (2014). Urban Underground Pipelines Mapping Using Ground Penetrating Radar. IOP Conference Series: Earth and Environmental Science, 18(1), 012167.

Jessie (2017). 8 Basic Experience Design Considerations For Mobile Apps. Retrieved 27/04/2019 from 
https://hackernoon.com/ux-ui-design-for-mobileappsd4e 079 adce 37

Li, W., Han, Y., Liu, Y., Zhu, C., Ren, Y., Wang, Y., and Chen, G. (2018). RealTime Location-Based Rendering of Urban Underground Pipelines. ISPRS Int. J. Geo-Information, 7, 32.

Meemim Inc. (2018). Measuring effectiveness of Augmented Reality in the locate services industry. Retrieved 24/10/2018 from https://www.vgis.io/2018/07/25/measuring-effectivenessof-augmentedreality-in-the-locate-services-industry/

Office of Asset. (2017). History of Office of Asset (UTM). Retrieved 20/11/2018 from http://assets.utm.my/

Pestov, A. (2018). Enhanced utility management with integration of GIS and augmented reality. Retrieved 26/09/2018 from https://www.geospatialworld.net/blogs/transforming-theindustry-with-esrigis-and-advanced-visualisation-tools/

Rees, E. V. (2018). Enhancing GIS with Augmented Reality. Retrieved 25/10/2018 from https://www.spar3d.com/blogs/allover-the-map/enhancing-gisaugmented-reality/

Schall, G., Méndez, E., Kruijff, E., Veas, E., Junghanns, S., Reitinger, B., and Schmalstieg, D. (2009). Handheld Augmented Reality for underground infrastructure visualisation. Personal Ubiquitous Comput., 13(4), 281-291. doi:10.1007/s00779-008-0204-5

The Canadian Common Ground Alliance (2018). Canadian Common Ground Alliance DIRT Report Estimates Damage to Buried Utilities Continues to Cost Canada At Least \$1 Billion Every Year. Retrieved from http://www.canadiancga.com/page$776157 / 6895057$

Technologies, U. (2019). Unity User Manual (2019.1). Retrieved 13/04/2019 from https://docs.unity3d.com/Manual/index.html

The Star. (2018, 19/11/2018). TM Internet services down due to multiple fibre cut incidents. The Star. Retrieved from https://www.thestar.com.my/news/nation/2018/11/19/tminternet -services-down-due-to-multiple-fibre-cutincidents/

The Sun Daily. (15/06/2017). Utility service providers urged to announce excavation works on online portal 'Call Before You Dig'. The Sun Daily. Retrieved from https://www.thesundaily.my/archive/utilityserviceproviders-urged-announce-excavation-worksonlineportal-call-you-dig-ETARCH453514 